\title{
Impacto do Programa Saúde da Família sobre indicadores de saúde da criança em municípios de grande porte da região Nordeste do Brasil
}

\author{
Impact of Health Family Program concerning child \\ health indicators in large towns from Brazilian \\ Northeast region
}

Angelo Giuseppe Roncalli 1

Kenio Costa de Lima 1

1 Universidade Federal do Rio Grande do Norte. Av. Sen. Salgado Filho 1787, Lagoa Nova, 59056-000, Natal RN. roncalli@terra.com.br
Abstract The present study carried out in the context of the Baseline Studies of the PROESF was aimed at evaluating the impact of the Family Health Program (PSF) on indicators for child health in cities with more than 100,000 inhabitants in the Brazilian Northeast. Four cities were investigated. In each one, twenty censual sectors were selected randomly from areas covered by the PSF and compared with twenty sectors selected from areas not covered by the PSF on the basis of socioeconomic criteria. In most cases, no significant differences were found between the areas covered and not covered by the PSF. The only difference found was a significantly lower rate of hospital admissions due to diarrhea but this was on account of the Program of Community Health Agents. The PSF exerted no additional effect on the reduction of this indicator. It was also observed that the way by which the program is implemented in each city interferes directly in the results. Thus, there is no basis for considering the PSF per se ineffective or not differing from other programs with regard to its health care patterns. An evaluation of the PSF would necessarily have to include an analysis of the way the program is implemented and conducted in each case, besides considering its general socioeconomical and political characteristics.

Key words Primary health care, Family Health Program, Family Health Program's evaluation, Children's health
Resumo O objetivo deste estudo dentro dos Estudos de Linha de Base do Proesf foi avaliar o impacto do Programa Saúde da Família sobre indicadores relacionados à saúde da criança em municípios com mais de 100 mil habitantes. Foram pesquisados quatro municípios na região Nordeste, e em cada um deles foram sorteados 20 setores censitários em áreas cobertas pelo PSF e emparelhados a outros 20 setores em áreas não cobertas pelo PSF, a partir de critérios socioeconômicos. Os resultados mostraram que, na maior parte dos indicadores, não se observam diferenças significativas entre os resultados de áreas cobertas e não cobertas pelo PSF, destacando-se apenas a redução na taxa de internação por diarréia. Em relação a esta, a redução significativa se deu às expensas do Programa de Agentes Comunitários de Saúde, não trazendo o PSF efeito adicional sobre a redução do indicador. Pôde-se observar, também, que o modo como o programa é implementado em cada município influencia diretamente nos resultados, de modo que não há subsídios claros para decretaro PSF "per si" pouco resolutivo e sem diferencial em seus padrões de assistência à saúde. Torna-se, portanto, necessário levar em conta a análise do contexto da implantação e condução do PSF, além de suas características mais gerais relativas ao campo socioeconômico e de políticas públicas.

Palavras-chave Atenção básica, Programa Saúde da Família, Avaliação do Programa Saúde da Família, Saúde da criança 


\section{Introdução}

Na primeira metade da década de 1990, mais precisamente em 1994, o Ministério da Saúde adotou em nível nacional o Programa de Saúde da Família (PSF)1. Seu principal propósito era a organização da prática da atenção à saúde sob novas bases, substituindo o modelo tradicional, fazendo a saúde chegar mais perto das famílias, a fim de que houvesse uma melhoria na qualidade de vida dos brasileiros. Essa estratégia incorpora e reafirma os princípios do Sistema Único de Saúde (SUS) e está estruturada a partir da Unidade de Saúde da Família (USF), que se propõe a organizar suas ações sob a égide da integralidade e hierarquização, territorialização e cadastramento da clientela, a partir de uma equipe multiprofissional. Draibe ${ }^{2}$ afirma que a adscrição territorial da clientela em conjunto com a opção pela unidade familiar constituem-se em duas das principais inovações do PSF. Ainda que não representem um avanço do SUS, são, pelo menos, uma correção de suas insuficiências.

A adoção da Estratégia Saúde da Família (ESF) parte do reconhecimento de que iniciativas de introdução de mudanças substantivas no setor saúde, a partir da implantação do SUS, têm resultados pouco perceptíveis na estruturação dos serviços de saúde, apesar de seus avanços, exatamente por não promover alterações significativas no modelo assistencial ${ }^{3}$. A implementação dessa estratégia, tida como eixo estruturante da atenção básica, embora sob mesma orientação macropolítica, vem produzindo experiências qualitativamente diferentes e, em muitos casos, reproduzindo o modelo tradicional em novas roupagens, como já ressaltaram Silva Jr. ${ }^{4}$ e Merhy \& Franco ${ }^{5}$.

Uma questão importante na implementação de todo e qualquer modelo assistencial ou mesmo estratégia de atenção, como é o caso do PSF, é o processo de avaliação e monitoramento. Este processo, segundo Silva Jr. \& Mascarenhas6, necessita ser complementado com a abordagem da pesquisa avaliativa na busca da percepção dos movimentos de mudanças na qualidade da atenção, no que se refere, principalmente, ao processo de trabalho das equipes na relação com as populações adscritas e à integralidade da atenção à saúde. Eles afirmam ainda que mais do que uma intervenção pontual de caráter avaliativo, a pesquisa avaliativa acrescenta instrumentos para pensar no cotidiano dos serviços, das práticas dos seus profissionais e da relação com a população, numa visão autocrítica e estimulante do protagonismo desses atores.

Através da avaliação de resultados, consegue-se pesquisar se determinados programas cumpriram ou estão cumprindo seus objetivos. De acordo com Draibe $^{2}$, este tipo de avaliação é também denominado de "avaliação de impacto". Os impactos referem-se às alterações ou mudanças efetivas na realidade sobre a qual um programa intervém e por ele são provocadas. Os indicadores de impacto devem ser, portanto, capazes de aferir os efeitos líquidos do programa sobre a população-alvo.

Baker7 afirma que a avaliação de impacto determina se o programa teve os efeitos projetados em indivíduos, lares e instituições, assim como se tais efeitos podem ser atribuídos, de fato, à intervenção do projeto. Para isso, a autora sugere que é preciso controlar o elemento contrafactual ou a contrapartida: "o que aconteceria se o projeto não existisse?”.

Desse modo, tentando incorporar novos e mais abrangentes elementos à problemática, $o$ objetivo deste estudo foi avaliar o impacto do PSF sobre indicadores relacionados à saúde $\mathrm{da}$ criança em quatro municípios com mais de 100 mil habitantes da região Nordeste.

\section{Metodologia}

\section{Tipo de estudo}

Este estudo é parte da pesquisa avaliativa de linha-base do Projeto de Expansão e Consolidação do Saúde da Família (Proesf), cujos municípios avaliados pertencem ao Lote III Região Nordeste, que compreende os estados do Ceará, Sergipe e Bahia, conforme determinação do Ministério da Saúde, sob a responsabilidade do Núcleo de Estudos em Saúde Coletiva da Universidade Federal do Rio Grande do Norte (Nesc-UFRN).

Trata-se de um ensaio comunitário em paralelo, quase-randomizado. Na tipologia clássica dos estudos epidemiológicos, constitui-se num estudo agregado do ponto de vista do seu tipo operativo, e cuja intervenção (implantação do PSF) já foi realizada em diferentes tempos, de acordo com as características dos municípios. Seus efeitos, portanto, foram avaliados no tempo atual, o que caracteriza um estudo longitudinal. 

experimental e controle, o primeiro critério de inclusão para selecionar os municípios a partir do universo foi a cobertura do PSF. Importante destacar que, pelas características da pesquisa, somente municípios com mais de 100 mil habitantes participaram da amostra.

Admitindo-se, como ponto de corte arbitrário, uma cobertura máxima de $70 \%$ e, consequentemente, a possibilidade de dispor de $30 \%$ da área para a constituição de grupos controle, apenas 7 dos 21 municípios que faziam parte do lote preencheram este critério. Desses, tomando como base o parâmetro proposto pelo termo de referência da pesquisa em relação ao porte populacional, foram selecionados quatro municípios.

\section{- Definição das unidades amostrais secundárias}

Uma condição fundamental para um estudo como este é a homogeneidade entre as áreas nas quais temos a intervenção e as áreas nas quais ela não se faz presente. Assim, de algum modo, são necessários dados secundários relativos às áreas cobertas e não cobertas, no sentido de agrupá-los e gerar critérios de emparelhamento das mesmas.

Uma unidade de análise que contém informações relevantes para este fim em todas as

áreas, independentemente de cobertura, é o setor censitário. Da totalidade de setores de cada um dos municípios, foram excluídos aqueles que estão parcialmente inseridos em áreas cobertas pelo PSF. Portanto, somente aqueles totalmente inseridos em áreas cobertas e não cobertas compuseram o universo a partir do qual foram estabelecidos os grupos.

Uma vez estabelecido o universo a ser incluído, foram sorteados 20 setores das áreas cobertas pelo PSF há pelo menos 12 meses, os quais compuseram as áreas sob intervenção. Para compor as áreas de não intervenção (controles), foi realizado um emparelhamento intencional a partir dos dados constantes nas bases de dados dos setores censitários. Estes dados são relativos às principais variáveis, as quais, sabidamente, exercem influência sob as variáveis de estudo, entre elas as de natureza socioeconômica. A partir da "Base de dados por setor censitário", disponibilizada pelo Instituto Brasileiro de Geografia e Estatística (IBGE) ${ }^{8}$, foram selecionadas algumas variáveis socioeconômicas, as quais compuseram um índice classificatório final, para que o emparelhamento pudesse ser realizado. No quadro 1 estas variáveis estão descritas mais detalhadamente.

Todas as variáveis possuem natureza quantitativa e escalas de medida distintas (anos de estudo, reais, percentual, etc.); e, para que pudessem compor um único índice agregado, tiveram que sofrer um ajuste a partir da seguinte fórmula:

indicador da $=\frac{\text { valor observado }- \text { valor mínimo }}{\text { variável } x}$
valor máximo - valor mínimo

\section{Quadro 1}

Descrição das variáveis utilizadas para a construção do indicador agregado.

\begin{tabular}{|c|c|c|}
\hline Variável & Natureza & Descrição \\
\hline Escolaridade & Educacional & $\begin{array}{l}\text { Média de anos de estudo do chefe da família contados a partir do lo ano } \\
\text { do ensino fundamental }\end{array}$ \\
\hline Analfabetismo em adultos & Educacional & $\%$ de responsáveis por domicílios particulares permanentes não-alfabetizados \\
\hline Analfabetismo infantil & Educacional & $\%$ de indivíduos de 5 anos analfabetos \\
\hline Renda média & Econômica & $\begin{array}{l}\text { Rendimento nominal mensal médio do setor (em reais) relativo aos chefes } \\
\text { de domicílios particulares permanentes }\end{array}$ \\
\hline Desemprego & Econômica & $\%$ de responsáveis por domicílios particulares permanentes sem rendimento \\
\hline Pobreza & Econômica & $\begin{array}{l}\% \text { de responsáveis por domicílios particulares permanentes com renda } \\
\text { até meio salário mínimo }\end{array}$ \\
\hline Acesso à água tratada & Sanitária & \% de domicílios com água fornecida por rede geral \\
\hline Instalações sanitárias & Sanitária & $\%$ de domicílios sem banheiro \\
\hline Lixo & Sanitária & $\%$ de domicílios com lixo coletado \\
\hline
\end{tabular}


Cada variável passou, portanto, a ter um valor entre 0 e 1 , tornando-se mais estável. A soma de todos os valores das nove variáveis compôs, então, o índice geral de condição socioeconômica do setor. Considerando-se que algumas variáveis apresentam melhores condições para maiores valores, e outras o contrário, todas foram ajustadas para que, ao final, o índice representasse as melhores situações a partir dos maiores valores. Desse modo, os valores das variáveis relativas ao analfabetismo, desemprego, pobreza e instalações sanitárias foram invertidos. O índice geral de condição socioeconômica possui, portanto, a seguinte expressão:

Índice Geral $=$ Escolaridade + (1/(1-Analfabetismo em adultos $))+(1 /(1-$ Analfabetismo infantil $))+$ Renda média $+(1 /(1$-Desemprego $))$ $+(1 /(1$-Pobreza $))+$ Acesso à água + (1/(1-Instalações sanitárias) ) + Lixo

Com o Índice Geral obtido para cada um dos setores, o mesmo foi categorizado, a partir da distribuição percentil, em quatro faixas: Muito Baixo, Baixo, Médio e Alto. Uma vez estabelecida a classificação, este critério foi utilizado para o emparelhamento dos setores cobertos e não cobertos (experimentais e controles). Para cada setor sorteado do grupo experimental, foi escolhido um setor não coberto de mesmo escore, que serviu de controle. Desse modo, ficou garantido o controle dos vieses relativos às variáveis socioeconômicas.

\section{Variáveis}

A variável de interesse explanatória é a presença do PSF na área. Entre as variáveis de resposta (dependentes) estão aquelas relativas à situação de saúde e que possuem a capacidade de serem fortemente influenciadas pela presença do PSF. Estas variáveis estão descritas no quadro 2.

As variáveis independentes de confusão estão relacionadas aos distintos níveis de análise, considerando que foram obtidas em nível da família (domicílio), do setor censitário e do município. O quadro 3 ilustra a descrição das variáveis que foram utilizadas na análise.

Outras variáveis socioeconômicas relativas ao nível domiciliar foram também investigadas, entre elas a renda familiar mensal e a densidade domiciliar (número de pessoas por cômodo). Estas duas variáveis não foram incluídas no plano de análise pelo fato de apresentarem forte colinearidade com a escolaridade da mãe. Considerando ser esta última mais con- fiável, uma vez que dados de renda apresentam alto viés de informação, optou-se por utilizar a escolaridade da mãe como proxy de situação socioeconômica em nível domiciliar.

\section{Tamanho da amostra}

Para o cálculo do tamanho da amostra deste estudo, tomou-se como base a taxa de internação por Infecção Respiratória Aguda (IRA) em menores de 5 anos. Este indicador foi adotado para o cálculo do tamanho da amostra, em virtude de o mesmo ser o mais discriminatório da intervenção PSF, entre aqueles propostos para avaliação do impacto.

O tamanho da amostra para estudos de intervenção é calculado a partir do efeito presumido da intervenção (em nosso caso, a implantação do PSF) associado a determinados valores de $\alpha$ (confiança) e 1- $\beta$ (poder) ${ }^{9}$. Assumiuse que uma eventual diferença no citado indicador entre 2000 e 2004 pode, em parte, ser creditada à intervenção. Para efeito de cálculo da amostra, foram utilizados os indicadores referentes a 2000 e 2004 do estado a qual pertence o município, para que a diferença percentual pudesse ser obtida. Esse percentual de redução foi, então, aplicado ao indicador para o ano 2000 do município em estudo, para que fosse obtida a diferença padronizada.

\section{Instrumentos e estratégias de coleta de dados}

Foi desenvolvida uma ficha a ser aplicada pelos Agentes Comunitários de Saúde (ACS) nos domicílios. Este questionário-entrevista foi aplicado junto ao informante mais qualificado, sendo, portanto, um instrumento que agrega informações relativas a todos os residentes no domicílio.

Os ACS foram treinados em oficinas de trabalho de 12 horas de duração (três turnos), associando a familiarização com os instrumentos a um treinamento em campo.

\section{Análise dos dados}

O banco de dados da pesquisa foi construído na plataforma SPSS versão 10.0, com posterior verificação de consistência da digitação.

Considerando o modelo teórico do estudo, as variáveis independentes e os desfechos foram devidamente trabalhados no sentido de comporem um modelo de análise estratificada. 
Quadro 2

Descrição das variáveis dependentes (resposta) para o estudo de impacto.

\begin{tabular}{|c|c|}
\hline Variáveis & Descrição \\
\hline Cobertura vacinal para DPT em menores de 1 ano & $\begin{array}{l}\text { Proporção de crianças menores de } 1 \text { ano com esquema vacinal } \\
\text { para DPT adequado }\end{array}$ \\
\hline Taxa de internação por IRA em menores de 5 anos & $\begin{array}{l}\text { Proporção de crianças com menos de } 5 \text { anos de idade que nos últimos } \\
12 \text { meses foram admitidas em hospitais por causa de IRA }\end{array}$ \\
\hline Taxa de internação por diarréia em menores de 5 anos & $\begin{array}{l}\text { Proporção de crianças com menos de } 5 \text { anos de idade que nos últimos } \\
12 \text { meses foram admitidas em hospitais por causa de diarréia }\end{array}$ \\
\hline Mortalidade em menores de 1 ano & Proporção de óbitos em menores de 1 ano de idade \\
\hline Prevalência de baixo peso ao nascer & $\begin{array}{l}\text { Proporção de nascimentos com menos de } 2.500 \text { gramas ocorridos } \\
\text { nos últimos } 24 \text { meses }\end{array}$ \\
\hline
\end{tabular}

Legenda: DPT = Difteria, Coqueluche e Tétano; IRA = Infecção Respiratória Aguda.

\section{Quadro 3}

Descrição das variáveis independentes de confusão para o estudo de impacto.

\begin{tabular}{|c|c|}
\hline Variáveis & Descrição \\
\hline \multicolumn{2}{|l|}{ Obtidas em nível da família } \\
\hline Escolaridade da mãe & $\begin{array}{l}\text { Anos de estudo da mãe, dicotomizados a partir da mediana, formando as categorias } \\
\text { "baixa" e "alta". }\end{array}$ \\
\hline Tempo de cobertura do programa & $\begin{array}{l}\text { Número de anos em que o programa (PACS ou PSF) atua na área em que se situa } \\
\text { o domicílio. Posteriormente categorizado a partir da mediana em "baixo" e "alto", } \\
\text { considerando cada programa especificamente (PACS e PSF). }\end{array}$ \\
\hline \multicolumn{2}{|l|}{ Obtidas em nível de setor censitário } \\
\hline Classificação do setor & $\begin{array}{l}\text { Classificação do setor definida pelo indicador agregado (ver quadro 1). As } 4 \text { categorias } \\
\text { foram transformadas em apenas duas, sendo a primeira categoria ("muito baixa") } \\
\text { definida como "desfavorável" e a associação das outras três ("baixa", "moderada" } \\
\text { e "alta") definida como "favorável". }\end{array}$ \\
\hline \multicolumn{2}{|l|}{ Obtidas em nível municipal } \\
\hline Classificação do município & $\begin{array}{l}\text { Classificação do município com relação ao desempenho do mesmo nos indicadores } \\
\text { isoladamente. Em cada indicador, os municípios foram agrupados em "favorável" } \\
\text { e "desfavorável". }\end{array}$ \\
\hline
\end{tabular}

Neste sentido, os efeitos da intervenção, ajustados para todas as variáveis de confusão, foram observados nos principais desfechos a partir do cálculo da razão de prevalência ajustada para cada estrato e seus respectivos intervalos de confiança. Considerou-se como nível de significância para este estudo o limite de $\alpha=0,05$.

\section{Resultados e discussão}

Os dados, originalmente obtidos por domicílio, foram transformados em dados por indivíduo, totalizando 24.695 pessoas.

A taxa de perda por recusa foi baixa, tendo os dados sido coletados com sucesso em cerca de $95 \%$ dos casos. Essas perdas também ocorreram de modo uniforme entre as distintas áreas. Com relação à amostra de interesse, a partir da qual foi calculado o tamanho, foram encontradas 2.144 crianças menores de 5 anos. Este total correspondeu a $8,9 \%$ dos indivíduos pesquisados, um percentual um pouco abaixo do esperado (11,41\%). Também corresponde a $52,2 \%$ da amostra exigida de 4.104 indivíduos, situando-se abaixo da margem mínima de $80 \%$ (uma vez que foi estimada uma perda de 20\%).

Importante destacar que o agravo escolhido como base para o cálculo da amostra possui, entre os vários indicadores, uma prevalência situada entre as mais altas, de modo que o tamanho amostral obtido para este indicador 
não necessariamente cumpre a mesma tarefa de significância para outros indicadores de menor prevalência. $\mathrm{O}$ tamanho da amostra calculado considerou tanto a relevância do indicador, como sendo representativo das ações de saúde em nível de PSF, quanto a factibilidade do estudo.

\section{Cobertura de vacinação para DPT em crianças menores de 1 ano}

Das variáveis independentes analisadas, apenas a classificação socioeconômica, avaliada a partir da escolaridade da mãe, apresentou associação com a proporção de crianças menores de um ano sem cobertura vacinal para DPT, embora tal associação tenha se situado no limiar da significância estatística. Nesse sentido, famílias cujas mães tinham baixa escolaridade apresentaram uma maior proporção de crianças menores de um ano sem cobertura vacinal para DPT, na ordem de 1,7 vez. Portanto, essa variável pode ter influenciado o efeito da variável independente de interesse (cobertura do programa), e assim o fato de estar coberto ou não pelo PSF, PACS ou ambos se apresentar "contaminado" pela condição socioeconômica das famílias.

Sendo assim, realizou-se uma análise estratificada, tomando-se como base a cobertura do programa para famílias cujas mães tiveram baixa e alta escolaridade, assim como no todo. Os dados podem ser observados na tabela 1 .

A despeito de a condição socioeconômica ter influenciado na proporção de crianças menores de um ano sem cobertura vacinal, esta influência não foi verificada sobre a variável cobertura do programa (Tabela 1). Logo, independentemente da condição socioeconômica, nenhum dos programas - PACS, PSF e ambos associados - exerceu impacto sobre a cobertura vacinal para DPT em crianças menores de um ano.

Provavelmente, por ser a cobertura vacinal para DPT um atributo dos programas de imunização em nível nacional e, portanto, adotados de modo semelhante em áreas sem cobertura de PACS e PSF, estes não tenham tido impacto, além do que já é habitual em áreas co-

\section{Tabela 1}

Comparação da proporção de crianças menores de um ano sem cobertura vacinal para DPT entre as categorias das variáveis independentes de interesse estratificadas por condição socioeconômica.

\begin{tabular}{|c|c|c|c|c|}
\hline \multirow[t]{2}{*}{ Variável independente } & \multicolumn{4}{|c|}{ Crianças menores de um ano sem cobertura vacinal para DPT } \\
\hline & Proporção & $\mathrm{RP}$ & IC (95\%) & $p$ \\
\hline \multicolumn{5}{|l|}{$\begin{array}{l}\text { Condição socioeconômica } \\
\text { desfavorável }\end{array}$} \\
\hline \multicolumn{5}{|l|}{ Tipo de cobertura } \\
\hline Sem cobertura & $(4 / 45)$ & 1 & & \\
\hline Cobertura PACS & $13,1 \% \quad(8 / 61)$ & 0,72 & $0,23-2,25$ & 0,758 \\
\hline Cobertura PSF & $12,2 \% \quad(28 / 230)$ & 0,73 & $0,23-2,11$ & 0,708 \\
\hline Cobertura PACS e PSF & $12,4 \% \quad(36 / 291)$ & 0,72 & $0,27-1,92$ & 0,672 \\
\hline \multicolumn{5}{|l|}{$\begin{array}{l}\text { Condição socioeconômica } \\
\text { favorável }\end{array}$} \\
\hline \multicolumn{5}{|l|}{ Tipo de cobertura } \\
\hline Sem cobertura & $8,0 \% \quad(4 / 50)$ & 1 & & \\
\hline Cobertura PACS & $10,4 \% \quad(8 / 77)$ & 0,77 & $0,24-2,42$ & 0,763 \\
\hline Cobertura PSF & $6,2 \% \quad(12 / 193)$ & 1,29 & $0,43-3,82$ & 0,894 \\
\hline Cobertura PACS e PSF & $7,4 \% \quad(20 / 270)$ & 1,08 & $0,39-3,03$ & 0,883 \\
\hline \multicolumn{5}{|l|}{ Total } \\
\hline \multicolumn{5}{|l|}{ Tipo de cobertura } \\
\hline Sem cobertura & $8,4 \% \quad(8 / 95)$ & 1 & & \\
\hline Cobertura PACS & $11,6 \% \quad(16 / 138)$ & 0,75 & $0,33-1,67$ & 0,619 \\
\hline Cobertura PSF & $9,5 \% \quad(40 / 423)$ & 0,93 & $0,45-1,92$ & 0,906 \\
\hline Cobertura PACS e PSF & $10,0 \% \quad(56 / 561)$ & 0,86 & $0,42-1,75$ & 0,774 \\
\hline
\end{tabular}

Legenda: DPT = Difteria, Coqueluche, Tétano; RP = Razão de Prevalência; IC = Intervalo de Confiança;

PACS $=$ Programa Agentes Comunitários de Saúde; PSF = Programa Saúde da Família. 
bertas por unidades tradicionais de saúde. Há que se destacar aqui o reduzido tamanho da amostra, sobretudo em áreas sem cobertura de programa algum. Isso, indubitavelmente, pode ter interferido nos resultados.

Cruz ${ }^{10}$, em seu trabalho no estado de São Paulo, ao estudar cobertura vacinal e taxa de abandono da vacina DPT em municípios com e sem a presença de PSF, observou que a implantação desse programa foi um dos fatores que contribuíram para a queda das taxas de abandono ao esquema vacinal para DPT. Do mesmo modo, Silva11, ao avaliar a evolução de indicadores relacionados à saúde da criança no município de Sobral (CE), chama a atenção para o fato de que em cinco anos de observação pós-implantação do PSF, a imunização em crianças menores de 5 anos ascendeu quase $30 \%$. O mesmo destaque ao aumento da cobertura vacinal foi dado por Moura et. al12, ao estudarem a atenção pré-natal ofertada em uma microrregião de saúde do Ceará assistida por equipes do PSF.

Ressalvas nas comparações com os resultados deste estudo devem ser feitas. Inicialmente os trabalhos realizados no estado do Ceará, além de utilizarem metodologia diferente, sendo estudos do tipo antes-depois, foram realizados em áreas onde a implantação do PSF se deu a contento e já está bem consolidada, sendo Sobral, por exemplo, uma referência em termos de organização da assistência à saúde. No que se refere ao estudo de Cruz ${ }^{10}$, este foi desenvolvido em municípios paulistas, onde as condições socioeconômicas e sanitárias são bem mais favoráveis que os ora analisados.

Quando da observação de áreas cobertas unicamente pelo Programa Saúde da Família, tomando-se os municípios individualmente, também não se observaram diferenças significativas na proporção de crianças menores de um ano que não foram vacinadas para DPT em relação a áreas não cobertas pelo PSF.

Para se analisar o efeito do modelo assistencial implementado, observou-se o comportamento da variável em função da condição do município do ponto de vista do seu resultado específico para o indicador. Neste caso, tanto municípios com condição favorável quanto desfavorável para este indicador apresentaram resultados semelhantes para áreas cobertas e não cobertas.

Isso reforça o fato de que o PSF nos municípios analisados não vem apresentando melhorias na cobertura vacinal por DPT, além do que já é feito através dos programas de imunização. Vale salientar que se trata de um estudo de linha base e que, com a consolidação do referido programa, possíveis impactos sejam encontrados, como já demonstrado em outros estudos.

\section{Óbitos em menores de 1 ano}

Das variáveis testadas, apenas a condição do município analisado interferiu na prevalência de óbitos em crianças menores de um ano. Município considerado desfavorável apresentou maior prevalência de óbitos em crianças menores de um ano na ordem de 3,12 vezes. A condição do município, no entanto, apesar de apresentar associação significativa com a prevalência de óbitos, não teve sua análise estratificada realizada em relação a esta variável dependente. Isto porque se acredita que a classificação do município em relação à implantação do PSF seja uma variável explanatória do que poderia ser observado em relação à cobertura ou não pelo PSF, isoladamente ou combinado com o PACS.

Sendo assim, a variável cobertura do programa foi analisada sem que tais variáveis pudessem estar contaminando o efeito da cobertura do programa. Tais dados podem ser observados na tabela 2 .

Como se pode observar, o PSF isoladamente, ou associado ao PACS, não exerceu impacto significativo sobre a proporção de óbitos em menores de um ano.

Óbitos em menores de um ano refletem, entre outros aspectos, a cobertura assistencial às mães através das consultas de pré-natal. Outros resultados obtidos por esta pesquisa demonstram que o PSF não exerceu impacto positivo sobre a cobertura pelo pré-natal. Sendo assim, era esperado que os óbitos em menores de um ano também não tivessem sofrido influência dos programas PACS e PSF. Como a assistência pelo pré-natal tem se tornado de grande abrangência, independentemente da existência do PSF, seu efeito adicional não pôde ser captado neste estudo.

Ao contrário, em Sobral (CE), no estudo de Silva11, a redução da taxa de mortalidade infantil foi significativa em sete anos de implantação do PSF, com redução da mortalidade neonatal e pós-neonatal. Segundo a autora, óbitos infantis no domicílio foram reduzidos em dois pontos percentuais em sete anos. Resultados similares foram encontrados no estudo de Cruz ${ }^{10}$ no estado de São Paulo. 


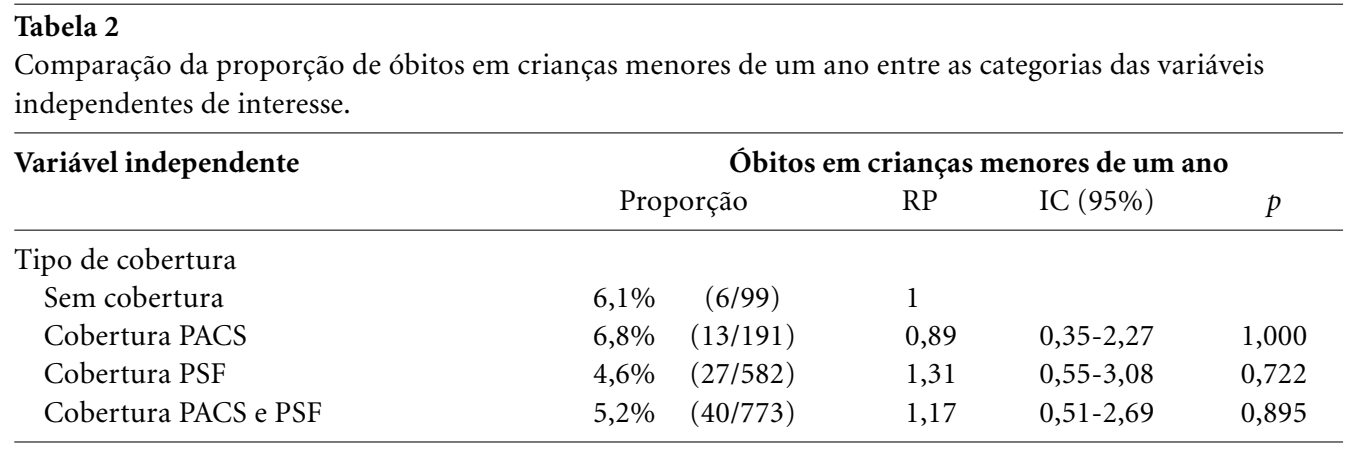

Legenda: RP = Razão de Prevalência; IC = Intervalo de Confiança; PACS = Programa Agentes Comunitários de Saúde; PSF $=$ Programa Saúde da Família.

Uma das justificativas para as possíveis diferenças entre os estudos, além das já discutidas para a cobertura vacinal para DPT, foi que a proporção de óbitos em todas as situações deste estudo foi baixa, necessitando de uma amostra significativamente maior para que pudéssemos verificar qualquer efeito dos programas em questão.

Quando se considera o "efeito município", observa-se que este indicador é também influenciado pelo modo como o modelo é implantado. Municípios classificados como "desfavorável” em relação a este indicador apresentam taxas significativamente maiores que os classificados favoravelmente $(p=0,040)$, denotando que as características de implantação podem influenciar no impacto sobre indicadores.

\section{Taxa de internação por IRA em crianças menores de 5 anos}

Do mesmo modo que para a cobertura vacinal para DPT, a única variável que apresentou associação com a taxa de internação de crianças menores de 5 anos por IRA foi a condição socioeconômica da família a que tal criança pertence. Famílias cujas mães possuíam baixa escolaridade apresentaram significativamente uma maior taxa de internação por IRA. Tais crianças apresentaram uma chance $1,3 \mathrm{vez}$ maior de terem sido internadas por IRA do que crianças cujas mães apresentaram escolaridade alta.

Sendo assim, a possível influência dessa variável no efeito da cobertura do programa em relação à taxa de internação por IRA encontrase expressa na tabela 3.

De acordo com a tabela 3, pode-se observar que o PSF não exerceu impacto sobre a taxa de internação por IRA nem em famílias com bai- xa, nem com alta escolaridade, isoladamente ou associado ao PACS.

No entanto, destaca-se que em áreas cobertas unicamente pelo PACS, a taxa de internação por IRA foi significativamente menor que em áreas sem cobertura por programa algum, e que este efeito foi perdido quando se incorporou o PSF para realização da análise. Tal efeito, como visto na tabela 3 , foi influenciado pela condição socioeconômica das famílias, sendo significativo apenas naquelas famílias com condição socioeconômica favorável. Nestas, a taxa de internação por IRA foi de $13,3 \%$, em detrimento de $22,9 \%$ de áreas não cobertas. Nesse sentido, crianças menores de 5 anos de famílias com condição socioeconômica favorável, residentes em áreas não cobertas pelo PACS, têm chance 1,9 vez maior de serem internadas por IRA.

É importante considerar que a taxa de internação por IRA reflete uma falta de atenção preventiva e curativa aos pacientes com tal agravo e, conseqüentemente, um estrangulamento da atenção básica, que deixa a desejar em relação à sua resolutividade. Sendo assim, os pacientes acabam nos hospitais para que a infecção seja debelada.

Este fato pode ser ilustrado no estudo de Cruz ${ }^{10}$ no estado de São Paulo, que concluiu que a implantação do PSF nos municípios analisados não contribuiu para a queda nas taxas de hospitalização, do mesmo modo que neste estudo em relação especificamente à IRA. Ao contrário, o estudo de Silva11, em Sobral (CE), afirma que a partir de 2001 não houve nenhum registro de óbito por infecção respiratória agu$\mathrm{da}$, fato este creditado à organização da atenção à saúde, em especial ao Programa Saúde da Família. 
Tabela 3

Comparação da taxa de internação por IRA em menores de 5 anos entre as categorias das variáveis independentes de interesse estratificadas por condição socioeconômica.

\begin{tabular}{|c|c|c|c|c|}
\hline \multirow[t]{2}{*}{ Variável independente } & \multicolumn{4}{|c|}{ Internação por IRA em menores de 5 anos } \\
\hline & Proporção & $\mathrm{RP}$ & IC $(95 \%)$ & $p$ \\
\hline \multicolumn{5}{|l|}{$\begin{array}{l}\text { Condição socioeconômica } \\
\text { desfavorável }\end{array}$} \\
\hline \multicolumn{5}{|l|}{ Tipo de cobertura } \\
\hline Sem cobertura & $22,4 \% \quad(22 / 98)$ & 1 & & \\
\hline Cobertura PACS & $18,5 \% \quad(43 / 233)$ & 1,22 & $0,77-1,92$ & 0,494 \\
\hline Cobertura PSF & $20,9 \% \quad(158 / 755)$ & 1,07 & $0,72-1,59$ & 0,829 \\
\hline Cobertura PACS e PSF & $20,3 \% \quad(201 / 988)$ & 1,10 & $0,75-1,63$ & 0,718 \\
\hline \multicolumn{5}{|l|}{$\begin{array}{l}\text { Condição socioeconômica } \\
\text { favorável }\end{array}$} \\
\hline \multicolumn{5}{|l|}{ Tipo de cobertura } \\
\hline Sem cobertura & $22,9 \% \quad(27 / 118)$ & 1 & & \\
\hline Cobertura PACS & $13,3 \% \quad(33 / 248)$ & 1,72 & $1,09-2,72$ & 0,031 \\
\hline Cobertura PSF & $17,9 \% \quad(115 / 642)$ & 1,28 & $0,88-1,85$ & 0,253 \\
\hline Cobertura PACS e PSF & $16,6 \% \quad(148 / 890)$ & 1,38 & $0,96-1,98$ & 0,120 \\
\hline \multicolumn{5}{|l|}{ Total } \\
\hline \multicolumn{5}{|l|}{ Tipo de cobertura } \\
\hline Sem cobertura & $22,7 \% \quad(49 / 216)$ & 1 & & \\
\hline Cobertura PACS & $15,8 \% \quad(76 / 481)$ & 1,44 & $1,05-2,00$ & 0,037 \\
\hline Cobertura PSF & $19,5 \%(273 / 1.397)$ & 1,17 & $0,90-1,54$ & 0,325 \\
\hline Cobertura PACS e PSF & $18,6 \%(349 / 1.878)$ & 1,24 & $0,95-1,61$ & 0,173 \\
\hline
\end{tabular}

Legenda: RP = Razão de Prevalência; IC = Intervalo de Confiança; PACS = Programa Agentes Comunitários de Saúde; PSF $=$ Programa Saúde da Família.

A influência do modelo de implantação também esteve presente neste indicador, uma vez que as taxas de internação por IRA em menores de 5 anos são significativamente maiores em municípios classificados como "desfavorável” $(\mathrm{p}=0,001)$.

\section{Taxa de internação por diarréia em crianças menores de 5 anos}

Nenhuma das variáveis de confusão testadas apresentou associação significativa com o desfecho taxa de internação por diarréia em crianças menores de 5 anos. Sendo assim, qualquer efeito observado neste indicador seria devido à cobertura ou não do PSF isoladamente, em conjunto com o PACS, ou mesmo só do PACS. Tal efeito pode ser observado na tabela 4.

De acordo com os dados apresentados, a taxa de internação por diarréia em crianças menores de 5 anos foi significativamente menor nos municípios estudados em áreas com cobertura de algum programa, situando-se em $11,0 \%$, em detrimento aos 19,4\% em áreas não cober- tas por nenhum programa. Nestas, as crianças menores de 5 anos teriam quase duas vezes mais chances de serem internadas por diarréia do que aquelas residentes nas demais áreas não cobertas. Para esse indicador, ambos os programas exerceram impacto sobre este agravo; entretanto não há diferenças no impacto entre eles.

A taxa de internação por diarréia é um indicador sensível a qualquer ação preventiva e curativa básica. Diarréia é um agravo de fácil diagnóstico e muitas vezes resolvido em nível domiciliar, quer seja por cuidados próprios das famílias ou, mais especificamente, de assistência curativa básica em nível de família ou de unidade de saúde. O acompanhamento das famílias através de visitas domiciliares pelos ACS parece ser suficiente para que se evite uma internação por esse agravo. Sendo assim, em áreas cobertas por ACS, e onde os mesmos sejam atuantes, imagina-se que haja um efetivo impacto sobre sua taxa de internação, o que foi observado nesse estudo em relação aos dois programas, PACS e PSF. Não foram observadas diferenças significativas quando foram compara- 
dos os grupos de municípios com condição favorável e desfavorável com relação ao indicador $(\mathrm{p}=0,505)$.

\section{Baixo peso ao nascer}

Nenhuma das variáveis confundidoras analisadas apresentou associação significativa com a prevalência de nascimentos de crianças com baixo peso. Nesse sentido, o efeito líquido da cobertura do programa pode ser visto e analisado na tabela 5 .

Nenhum efeito significativo pôde ser observado em relação a esse agravo em áreas cobertas pelo PSF isoladamente, nem associado ao PACS, quando comparadas a áreas não cobertas por nenhum programa. Há que se ressaltar que o PACS isoladamente também não exerceu impacto sobre esse agravo, provavelmente pela baixa prevalência de crianças de baixo peso mesmo entre aquelas nascidas em áreas descobertas, denotando a universalização dos programas de pré-natal desenvolvidos nessas áreas nas Unidades Básicas de Saúde (UBS), uma vez que a maior parcela de responsabilidade para a prevenção do nascimento de crianças de baixo peso se deve a um pré-natal bem conduzido. Conforme outros resultados obtidos por esta pesquisa, a cobertura pelo PSF não exerceu impacto sobre o aumento de cobertura do prénatal nos municípios analisados em conjunto. Logo, não seria surpresa que o PSF também não tenha exercido impacto sobre a proporção de nascimentos de crianças com baixo peso.

Tais dados se contrapõem aos estudos até então publicados sobre a influência da implantação do PSF no pré-natal e, conseqüentemente, na proporção de nascimento de crianças de baixo peso. Em geral, os estudos avaliam como de impacto positivo a implantação do PSF sobre o número de consultas 11,12 e a qualidade do pré-natal13, 14 .

Tendo como base o efeito do município neste indicador, observou-se uma ausência de significância estatística na diferença entre as proporções observadas nos municípios classificados como "favorável" e "desfavorável" $(\mathrm{p}=0,342)$.

Tabela 4

Comparação da taxa de internação por diarréia em menores de 5 anos entre as categorias das variáveis independentes de interesse.

\begin{tabular}{|c|c|c|c|c|}
\hline \multirow[t]{2}{*}{ Variável independente } & \multicolumn{4}{|c|}{ Internação por diarréia em menores de 5 anos } \\
\hline & Proporção & $\mathrm{RP}$ & IC $(95 \%)$ & $p$ \\
\hline \multicolumn{5}{|l|}{ Tipo de cobertura } \\
\hline Sem cobertura & $19,4 \% \quad(42 / 216)$ & 1 & & \\
\hline Cobertura PACS & $11,0 \% \quad(53 / 481)$ & 1,76 & $1,22-2,56$ & 0,004 \\
\hline Cobertura PSF & $11,0 \%(154 / 1.400)$ & 1,77 & $1,30-2,41$ & 0,001 \\
\hline Cobertura PACS e PSF & $11,0 \%(207 / 1.881)$ & 1,77 & $1,31-2,39$ & $<0,001$ \\
\hline
\end{tabular}

Legenda: RP = Razão de Prevalência; IC = Intervalo de Confiança; PACS = Programa Agentes Comunitários de Saúde; PSF = Programa Saúde da Família.

\section{Tabela 5}

Comparação da proporção de nascimentos com baixo peso entre as categorias das variáveis independentes de interesse.

\begin{tabular}{lccccc}
\hline Variável independente & \multicolumn{5}{c}{ Nascimentos com baixo peso } \\
& \multicolumn{2}{c}{ Proporção } & OR & IC (95\%) & $p$ \\
\hline Tipo de cobertura & & & & & \\
$\quad$ Sem cobertura & $12,1 \%$ & $(12 / 99)$ & 1 & & \\
Cobertura PACS & $11,1 \%$ & $(21 / 190)$ & 1,10 & $0,56-2,14$ & 0,939 \\
Cobertura PSF & $10,0 \%$ & $(58 / 579)$ & 1,21 & $0,67-2,17$ & 0,648 \\
Cobertura PACS e PSF & $10,3 \%$ & $(79 / 769)$ & 1,18 & $0,67-2,09$ & 0,696 \\
\hline
\end{tabular}

Legenda: RP = Razão de Prevalência; IC = Intervalo de Confiança; PACS = Programa Agentes Comunitários de Saúde; PSF $=$ Programa Saúde da Família. 


\section{Considerações finais}

Os indicadores utilizados nesta pesquisa mostraram uma clara relação com os fatores socioeconômicos, medidos em nível domiciliar (escolaridade da mãe) e de setor censitário (indicador sócio-sanitário). No mais das vezes, áreas de situação socioeconômica desfavorável apresentaram os piores indicadores. Essa forma de análise é importante nesse tipo de estudo, uma vez que se procura captar o efeito líquido da intervenção (no caso, o PSF), e tais variáveis podem atuar como fatores de confusão. A associação entre estes eventos é esperada, sendo suficientemente discutida no campo dos estudos sobre a causação social dos problemas de saúde.

A análise dessas associações demonstrou que as mesmas não exerceram influência sobre o efeito da intervenção nos vários indicadores estudados, o que ficou demonstrado a partir da análise estratificada. Isto deixa claro que a estratégia metodológica de emparelhamento das áreas experimental e controle, baseada no indicador sócio-sanitário agregado para setor, deu conta de controlar esses vieses, assegurando validade ao estudo.

Depreende-se que o PSF, tomando como base os quatro municípios em conjunto, exerceu pouco impacto sobre os agravos estudados, destacando-se apenas a redução da taxa de internação por diarréia. Em relação a esta, também houve redução significativa proporcionada pelo PACS, não trazendo o PSF adicional de redução. Nas demais, em sua grande maioria, não houve diferença entre áreas cobertas e não cobertas pelo PSF, mesmo quando incorporado a esse último a cobertura pelo PACS.

Situações peculiares foram observadas quando da análise considerando o perfil do município em relação ao indicador estudado, denotando uma influência das características de im- plantação dos programas. Assim, é importante destacar que a ausência de impacto pode ser creditada, em grande parte, à maneira como os programas estão sendo implantados, de modo que não há subsídios claros para decretar o PSF “per se" pouco resolutivo e sem diferencial em seus padrões de assistência à saúde. Isso fica claro quando se observa o comportamento de determinado indicador na análise agregada e quando verificado em cada município isoladamente.

Assim, é preciso levar em conta a análise do contexto da implantação e condução do PSF nestes municípios, além de suas características mais gerais relativas ao campo socioeconômico e de políticas públicas.

Finalmente, cabe ressaltar que este estudo tem algumas limitações que impedem maiores generalizações, ou seja, sua validade externa é comprometida pelo universo estudado (quatro municípios de dois estados da região Nordeste). Contudo, alguns pontos analisados podem ser apropriados pelas diversas iniciativas de implantação do programa em todo o País, no sentido de não somente aperfeiçoá-lo, mas, fundamentalmente, reconhecer suas limitações. Ficou claro também, por alguns pontos aqui discutidos, que determinadas situações de saúde se apresentam como resultado das condições concretas de existência sobre as quais os serviços de saúde (quaisquer que sejam) possuem limitada capacidade de impacto. Neste sentido, há que se entender o campo da saúde como um constructo resultante de um conjunto de ações nas mais variadas áreas, como bem descreve o ideário constitucional da saúde.

Estudos subseqüentes que avaliem o impacto da implantação de programas devem ser continuamente realizados, incorporando a pesquisa avaliativa como um componente do modelo assistencial.

\section{Colaboradores}

AG Roncalli e KC Lima participaram igualmente de todas as etapas da elaboração do artigo. 


\section{Referências}

1. Brasil. Ministério da Saúde. Saúde da Família [acessado 2005 Sep 12]. Disponível em: http:// www.saude. gov.br/psf

2. Draibe SM. Avaliação de implementação: esboço de uma metodologia de trabalho em políticas públicas. In: Barreira MCR, Carvalho MCB, organizadores. Tendências e perspectivas na avaliação de políticas e programas sociais. São Paulo: IEE/PUC; 2004. p. 15-42.

3. Senna MCM. Eqüidade e política de saúde: algumas reflexões sobre o Programa Saúde da Família. Cad Saúde Pública 2002; 18(1):203-11.

4. Silva Jr. AG. Modelos tecnoassistenciais em saúde: o debate no campo da saúde coletiva. São Paulo: Hucitec; 1998.

5. Merhy E, Franco TB. Programa Saúde da Família: somos contra ou a favor? Saúde em Debate 2002; 26: 118-22.

6. Silva Jr. AG, Mascarenhas MTM. Avaliação da atenção básica em saúde sob a ótica da integralidade: aspectos conceituais e metodológicos. In: Alves DS, Guljor AG. Cuidado: as fronteiras da integralidade. São Paulo: Hucitec; Rio de Janeiro: Abrasco; 2004. p. 241-55.

7. Baker J. Avaliando o impacto de projetos em desenvolvimento voltados à pobreza. In: Barreira MCR, Carvalho MCB, organizadores. Tendências e perspectivas na avaliação de políticas e programas sociais. São Paulo: IEE-PUC; 2001. p. 59-76.

8. Brasil. Ministério do Planejamento, Orçamento e Gestão. Instituto Brasileiro de Geografia e Estatística. Base de dados por setor censitário [CD-ROM]. Rio de Janeiro: IBGE; 2005.
9. Altman DG. Practical statistics for medical research. New York: Chapman \& Hall, CRC; 1990.

10. Cruz MGB. PSF - Programa de Saúde da Família: comparando a mortalidade infantil, cobertura vacinal e hospitalizações, entre municípios com e sem o programa no Estado de São Paulo [dissertação]. São Paulo (SP): Faculdade de Saúde Pública da USP; 2002.

11. Silva AC. O impacto do Programa de Saúde da Família no município de Sobral-Ceará: uma análise da evolução de saúde das crianças menores de cinco anos de idade no período de 1995-2002 [tese]. São Paulo: Faculdade de Medicina da Universidade de São Paulo; 2003.

12. Moura ERF, Holanda Jr. F, Rodrigues MSP. Avaliação da assistência pré-natal oferecida em uma microrregião de saúde do Ceará, Brasil. Cad Saúde Pública 2003; 19(6):1791-9.

13. Marquetti JL. Mortalidade infantil X Programa de Saúde da Família, no município de Curitiba [monografia]. Curitiba: Universidade Federal do Paraná; 1999.

14. Moura ERF, Rodrigues MSP, Silva RM. Programa Saúde da Família: impacto na assistência pré-natal. Rev Chil Salud Publica 2003; 7(1):25-32.

Artigo apresentado em 27/02/2006

Aprovado em 31/03/2006

Versão final apresentada em 17/04/2006 